\title{
DESENVOLVIMENTO E VALIDAC̣ÃO DE UMA EQUAC̣ÃO DE PREDIC̣ÃO DA CARGA DE TRABALHO PARA O TREINAMENTO COM PESOS NO EXERCÍCIO DE SUPINO RETO, PARA HOMENS DE NÍVEL INTERMEDIÁRIO
}

\author{
DEVELOPMENT AND VALIDATION OF EQUATION OF PREDICTION THE LOAD \\ OF WORK FOR TRAINING WITH WEIGHTS IN THE BENCH PRESS EXERCISES, \\ FOR MEN OF INTERMEDIATE LEVEL
}

\author{
AUTORES \\ Michel Leonardo Ferreira Lima' \\ José Fernandes Filho ${ }^{2}$ \\ Estélio Henrique Martin Dantas ${ }^{2}$ \\ Paula Roquete Fernandes ${ }^{3,4}$ \\ Felipe José Aidar ${ }^{5,6}$ \\ Victor Machado Reis ${ }^{6}$ \\ 'Universidade Castelo Branco - UCB \\ - Rio de Janeiro - Brasil \\ ${ }^{2}$ Universidade Castelo Branco - UCB \\ - Rio de Janeiro - Brasil \\ ${ }^{3}$ Centro de Excelência em Avaliação Física \\ - Rio de Janeiro - Brasil \\ ${ }^{4}$ Universidade do Grande Rio - UNIGRANRIO \\ ${ }^{5}$ Corpo de Bombeiros Militar de Minas Gerais \\ - Belo Horizonte - Brasil \\ ${ }^{6}$ Universidade de Trás-os-Montes \\ e Alto Douro - UTAD - Vila Real - Portugal
}

DESENVOLVIMENTO E VALIDAÇÃO DE UMA EQUAÇÃO DE PREDIC̣ÃO DA CARGA DE TRABALHO PARA O TREINAMENTO COM PESOS NO EXERCICIO DE SUPINO RETO, PARA HOMENS DE NIVEL INTERMEDIÁRIO 4(1): $39-47$

\section{PALAVRAS-CHAVE}

validação; equação de predição e carga de trabalho com pesos.

\section{KEYWORDS}

validation; equation of prediction; load of work for training with weights

data de submissão Julho 2006

data de aceitação Outubro 2007
RESUMO

Fundamentação: Os profissionais que trabalham com musculação tem questionado as formas de avaliação da força dinâmica para a prescrição de exercícios. Objetivo: desenvolver e validar uma equação de predição da Carga de Trabalho para o Treinamento com Pesos (CTTP) para o exercício de supino horizontal com pesos livres, em praticantes de musculação recreacional de nível intermediário. Materiais e Métodos: amostra composta de 60 (sessenta) praticantes de musculação recreacional. Para avaliação antropométrica e da composição corporal foram utilizados: a massa corporal, estatura e a estimativa da massa corporal magra, da massa gorda e do percentual de gordura. Para a força máxima de membros superiores foi utilizado o teste de 1RM no exercício de supino horizontal, utilizando pesos livres. Resultados: os resultados foram: Uma Repetição Máxima (1RM] (75,5 $\pm 12,7 \mathrm{Kg}) ;$ idade (23 $\pm 3,15 \mathrm{anos})$; \%gordura $[8,3 \pm 3,0 \%)$; massa corporal $(71,1 \pm 13,0 \mathrm{Kg})$; estatura $[172,0 \pm$ $8,4 \mathrm{~m})$; massa corporal magra $[62,8 \pm$ $10,6 \mathrm{Kg}$ ) e massa gorda $[6,2 \pm 3,5 \mathrm{Kg}$ ). Discussão e Conclusão: foi possível desenvolver e validar uma CTTP para o exercício de supino reto, com um Erro Padrão de Estimativa [EPE] de $3,378 \mathrm{~kg}$ ) e $\mathbf{R}^{2}=0,98$.

\section{ABSTRACT}

Back Ground: The professionals who work with muscle exercise have questioned the forms of evaluation of the dynamic force for exercise prescription purposes. Objective: to develop and to validate an equation for prediction of the load during weight training in the exercise of horizontal bench press with free weights. Materials and Methods: the sample comprised 60 [sixty) subjects engaged in recreational muscle exercise programs. For anthropometric evaluation and body composition the following measures were used: body mass, stature, estimated lean body mass, estimate body fat mass and the percentage of fat. For the maximum force of upper members the Repetition Maximum test (1RM) in the exercise of horizontal bench press was used. Results: the main results were: 1RM $(75,5 \pm 12,7 \mathrm{Kg})$; age [23 $\pm 3,15 y e a r s) ; ~ \% f a t ~[8,3 \pm 3,0 \%]$; body mass $(71,1 \pm 13,0 \mathrm{Kg})$; stature $(172,0 \pm 8,4 \mathrm{~m}) ;$ lean body mass $(62,8 \pm 10,6 \mathrm{Kg})$ and fat body mass $[6,2 \pm 3,5 \mathrm{Kg}$. Discussion: it was possible to develop and to validate an equation of prediction of the load of work for weight training in the exercise of horizontal bench press, with a Standard Error of Estimation [SEE] of $3,378 \mathrm{~kg}$ ), and $R^{2}=0,98$. 


\section{INTRODUC̣ÃO}

Atualmente, nas academias de ginástica, o treinamento com pesos tem um papel importante não só com a finalidade da performance física ou esportiva, mas também como treinamento preventivo na promoção de um estilo de vida mais saudável, inclusive em adultos velhos $^{13}$.

0 treinamento com pesos não é uma modalidade esportiva e sim uma forma de preparação física utilizada por atletas e pela população em geral. Podendo ser utilizado em programas visando à reabilitação, o estímulo à saúde, a estética e o lazer ${ }^{15,19,24}$.

Baseado nestas afirmações, quando se utiliza o treinamento com pesos como estímulos à saúde, à estética e ao lazer, parece ainda existir uma dúvida no que diz respeito à definição da carga que o aluno deve utilizar em seu programa de exercícios. Os profissionais que trabaIham com musculação questionam as formas de avaliação da força dinâmica para a prescrição de exercícios $^{8,20}$.

Hoje em dia o teste de uma repetição máxima (1RM) aparece como uma das formas utilizadas de mensuração da força máxima para elaboração de programas de musculação bem como para a avaliação e comparação do aumento da força muscular. A sua utilização, na maioria das vezes ou quase sempre, é muito cansativo e demorado, podendo ser indesejável sua realização com certas populações existentes nas academias de ginástica como: idosos, hipertensos, entre outros ${ }^{4,21}$. Para isto utiliza-se equação de predição para chegar ao valor de 1RM sem medição direta. Sendo assim, uma das equações utilizadas como suporte teórico para este estudo afirma existir uma diminuição aproximada da força muscular de 2 a 2,5\% para cada repetição realizada, em no máximo vinte repetições".

De qualquer forma, estabelecer uma carga de treinamento através do teste de 1 RM é de suma importância para que o trabalho muscular atinja o objetivo proposto", mesmo não sendo o mais indicado, viável e confiável ${ }^{8}$. Em função disso, torna-se importante estabelecer critérios científicos de avaliação da carga para um ou mais exercícios específicos, operacionalizando e trazendo para o âmbito da academia uma forma segura e prática da avaliação da força dinâmica.

Desta forma, o objetivo do presente estudo centra-se em buscar, através de uma equação validada, predizer a Carga de Trabalho para o treinamento com Pesos (CTTP) sem a necessidade de se fazer uma avaliação de 1RM. Isto contribuirá para uma correta orientação sobre o qual deverá ser a carga de treinamento que $o$ aluno poderá utilizar como referência, no exercício de supino horizontal. Procura-se com isso estabelecer qualidade, eficiência e segurança no que diz respeito a estratificações da força máxima dinâmica de membros superiores.

\section{METODOLOGIA}

\section{Amostra}

A amostra foi composta por 60 sujeitos praticantes de musculação recreativa a mais de três meses, faixa etária entre 18 e 30 anos de idade, do sexo masculino.

O tempo de prática foi adotado como critério de elegibilidade, tendo em vista que até oito semanas do início da prática de atividades resistidas há uma tendência a uma fase adaptativa neural. Esta fase adaptativa pode influenciar os resultados quando avaliada principalmente a força máxima. Após este período, o indivíduo tende a meIhorar seus niveis coordenativos, sendo estes muitas vezes relacionados com o aumento da força máxima ${ }^{6,10,12}$.

Os atletas eram informados que sua participação no estudo era voluntária. Foi utilizado como critério de elegibilidade ter feito ou estar fazendo o uso de alguma substância que pudesse estar interferindo na performance da amostra. Esta pesquisa seguiu rigorosamente os critérios propostos pela resolução n. ${ }^{\circ}$ 196, de 10 de Outubro de 1996, do Conselho Nacional de Saúde, em concordância com a Declaração de Helsinki, 1975 e adendo de 2000, sendo submetido para aprovação no comitê de ética institucional.

\section{Instrumentos}

A massa corporal foi mensurada em uma balança Balança Antropometrica Digital até $300 \mathrm{Kg}$ da marca WELMY, de plataforma, com estadiomêtro metálico aferida e certificada pelo IPEM/INMETRO, com precisão de $0,1 \mathrm{~kg}$, e a estatura foi obtida em um estadiômetro com precisão de $0,1 \mathrm{~cm}^{11}$.

As medidas foram realizadas por um único avaliador com um adipômetro científico da marca Lange. A avaliação da composição corporal e antropometria aconteceram antes da realização do teste de 1RM e os alunos não realizaram nenhuma atividade física prévia.

Para o teste de 1 RM foram utilizados 3 aplicadores com prática em exercícios resistidos que denominados juízes, e que ajudaram assegurar a confiabilidade e a validade de nossos dados. 


\section{Procedimentos}

Todos os indivíduos foram medidos e pesados descalços, vestindo apenas uma sunga. A partir dessas medidas, o índice de massa corporal (IMC) foi determinado pelo quociente massa corporal/estatura ${ }^{2}$, sendo a massa corporal expressa em quilogramas $(\mathrm{kg}$ ) e a estatura, em metros (m).

A composição corporal foi determinada pela técnica de espessura de dobras cutâneas. Três medidas foram tomadas em cada ponto anatômico [abdômen, peitoral e coxa], em seqüência rotacional, do lado direito do corpo, sendo registrado o valor mediano.

Tais medidas foram realizadas por um único avaliador com um adipômetro científico da marca Lange, de acordo com as padrões definidos ${ }^{22}$. A gordura corporal relativa $[\%$ gordura] foi calculada pela fórmula de $\operatorname{Siri}^{23}$, a partir da estimativa da densidade corporal determinada pela equação envolvendo a espessura de três dobras cutâneas ${ }^{14}$.

A avaliação de 1 RM de membros superiores, foi realizado o exercício de supino horizontal com pesos livres ${ }^{1}$.

Para o teste de 1RM, cada sujeito iniciou as tentativas com um peso que acreditasse pudesse ser levantado somente uma vez usando o esforço máximo.

Foi adicionado então incrementos peso até se atingir a carga máxima que pudesse ser levantada uma única vez. Caso o praticante não conseguisse realizar nenhuma repetição, subtrairia 2,4 a 2,5\% da carga, utilizada no teste ${ }^{2}$.

Os sujeitos descansaram entre 3-5 minutos entre tentativas. No Supino Horizontal, durante o teste de 1RM, cada sujeito se colocou na posição supina, mantendo sua cabeça, om- bros, e nádegas em contato constante com o banco e alinhados. A planta dos pés de cada sujeito permaneceu inteiramente apoiada no assoalho durante a tentativa no Banco de Supino Horizontal. Cada sujeito para a tentativa, recebe ajuda de dois outros juízes que se colocaram lateralmente, um em cada extremidade da barra, para tirar a barra do suporte do banco, recebendo a barra no comprimento do braço com os cotovelos estendidos sendo que a posição inicial era determinada por um outro juíz situado atrás da cabeça na direção do banco, este denominado juíz principal. A barra foi abaixada até o peito em um ponto na linha dos mamilos ou próxima a esta linha, com variabilidade permitida de aproximadamente 1 a $2 \mathrm{~cm}$ no sentido céfalo-caudal. Quando a barra tocou no peito, o juíz principal dava um comando através de uma palma, e o sujeito deveria estender seus braços, retornando o peso para a posição inicial até ficar como os cotovelos estendidos. Uma vez que os braços estivessem completamente esticados, o juíz principal dava um comando, e os juízes laterais ajudavam o sujeito a retornar o peso para o suporte do banco.

Todos os sujeitos foram submetidos três sessões de testes de 1-RM, no exercício supino horizontal, com intervalo de 48 a 72 horas entre cada sessão, para avaliação da força muscular.

0 teste foi precedido por uma série de aquecimento [6 a 10 repetições], com aproximadamente $50 \%$ da carga a ser utilizada na primeira tentativa de cada teste de 1-RM. A testagem foi iniciada dois minutos após o aquecimento. Portanto, a carga registrada como 1-RM foi aquela na qual foi possivel ao indivíduo completar somente uma única repetição ${ }^{7}$. 0 intervalo de transição entre os exercícios foi de três a cinco minutos.

Vale ressaltar que a forma e a técnica de execução de cada exercício foram padronizadas e continuamente monitoradas na tentativa de garantir a qualidade das informações. Além disso, os sujeitos realizaram os testes sempre no mesmo período do dia e não praticaram exercícios físicos durante o período experimental.

\section{Estatística}

0 tratamento estatístico atendeu à proposta básica, para o presente trabalho, utilizando a estatística descritiva, no sentido de constituir-se em uma análise descritiva do grupo em questão, observando-se os conceitos descritivos estruturados em valores médios e seus derivados para as variáveis de cunho contínuo; e das distribuições de freqüências para as variáveis de cunho discreto definidas pelos dados colhidos, quanto às observações dos elementos submetidos ao protocolo do experimento.

Aplicou-se o teste Komogorov-Smirnov, para verificação da normalidade da amostra. A estatística inferencial que compõe o teste de hipóteses foi baseada na correlação utilizando um tratamento paramétrico ou não-paramétrico segundo o resultado de normalidade realizado. Após a correlação feita, foram verificadas e geradas as equações de predição, para posterior escolha da que melhor se coadunava ao objetivo proposto.

Objetivando-se a medição dos testes, o presente trabalho pautou-se em consonância às considerações básicas do tratamento estatístico, a fim de manter-se a cientificidade da pesquisa, em que se considere o nível de significância de $p<0,05$. 


\begin{tabular}{|c|c|c|c|c|c|c|c|}
\hline & 1RM & Idade & $\% G$ & MC & Estatura & МсM & MASGord \\
\hline $\mathbf{N}$ & 60 & 60 & 60 & 60 & 60 & 60 & 60 \\
\hline Média & 75,5 & 23 & 8,3 & 71,1 & 172,0 & 62,8 & 6,2 \\
\hline DP & 12,7 & 3,15 & 3,0 & 13,0 & 8,4 & 10,6 & 3,5 \\
\hline Mínimo & 60 & 18 & 4,3 & 56,8 & 159 & 48,4 & 2,52 \\
\hline Máximo & 106 & 30 & 17,4 & 101,2 & 190 & 86,1 & 15,61 \\
\hline $\begin{array}{l}\text { Komogorov- } \\
\text {-Smirnov sig.p }\end{array}$ & 0,02 & & 0,473 & 0,105 & 0,173 & 0,132 & 0,184 \\
\hline Resultado & RN & & AN & AN & AN & AN & AN \\
\hline
\end{tabular}

TABELA 1

Estatística descritiva da amostra selecionada para o desenvolvimento da equação de predição.

LEGENDA: \%G: Percentual de Gordura; MC: Massa corporal; MCM: Massa corporal magra; MASGord: massa gorda; AN: Aceita Normalidade; RN: Rejeita Normalidade.

Para o tratamento estatístico foi utilizado o programa SPSS for Windows versão 12.0 .

\section{RESULTADOS}

Em um primeiro momento, o tratamento estatístico utilizado na presente pesquisa foi dividido na estatística descritiva, na qual são apresentados os resultados relativos à média e suas medidas de dispersão do grupo que compõe o desenvolvimento da equação e as características do grupo que validou o modelo, onde se procurou estabelecer uma característica própria e uma maior homogeneidade do grupo em estudo (tabela 1). Em seguida, são apresentados os resultados referentes à estatística inferencial, a fim de saber a relevância que o estudo acrescenta para a comunidade acadêmica e especialista em treinamento da força muscular.

\begin{tabular}{|c|c|c|c|c|c|c|}
\hline & 1RM & PGO & MCOR & Estat & МСM & MASGor \\
\hline $\mathbf{N}$ & 12 & 12 & 12 & 12 & 12 & 12 \\
\hline Média & 62,3 & 5,9 & 60,2 & 165 & 54,3 & 3,6 \\
\hline D.P & 2,01 & 1,13 & 2,63 & 3,05 & 2,02 & 0,82 \\
\hline Mínimo & 60 & 4,3 & 56,8 & 159 & 50,9 & 2,52 \\
\hline Máximo & 64 & 8,5 & 65 & 169 & 58,9 & 5,53 \\
\hline $\begin{array}{l}\text { Komogorov- } \\
\text {-Smirnov sig.p }\end{array}$ & 0,20 & 0,83 & 0,29 & 0,95 & 0,52 & 0,85 \\
\hline Resultado & AN & AN & AN & AN & AN & AN \\
\hline
\end{tabular}

TABELA2

Estatística descritiva da amostra selecionada para validação da equação de predição. LEgenda: PGO: Percentual de Gordura; MCOR: Massa Corporal; MCM: Massa Corporal Magra; MASGord: Massa Gorda; KS: Kolmogorov Smirnov; AN: Aceita Normalidade.

E finalmente, o estudo será concluído, com base nas informações geradas, de acordo com o que foi discutido e apresentado no modelo desenvolvido, acrescentando sua aplicabilidade prática e recomendações pertinentes a ele.

Dos resultados acima, tem-se que as variáveis apresentam distribuições ditas normais, exceto a variável dependente, ou seja, o teste de 1RM que responde pela natureza do problema, no qual a heterogeneidade se faz importante para aumento da significância do processo de resolução da equação de predição.

Em seguida (tabela 2), são apresentados os resultados da amostra selecionada para a validação da equação gerada de acordo com o objetivo deste estudo.

Nota-se a partir dos dados que a amostra apresenta normalidade para as variáveis selecionadas.

Os resultados relativos à Estatística Inferencial, as quais correspondem as variáveis que foram selecionadas para o desenvolvimento da equação preditora são apresentados na tabela 3.

Dos resultados acima temos que, as variáveis selecionadas para o constructo do modelo no seu contexto de significância apresentam alto índice de confiabilidade, uma vez que os indicadores de existência de relações causais entre as variáveis independentes (MC e estatura) e variável dependente (1RM) apresentam valores com sig. $P<0,01$, para a aceitação do modelo preditor. Ainda ressalta-se que sendo o coeficiente do MC maior que o da estatura, orienta dizer que o mesmo apresenta uma relevância maior na composição final do resultado comparativamente às medidas da estatura, a massa corporal dando o conteúdo do estrato principal e a estatura fazendo os ajustes de sintonia fina. 
Para tanto, utilizou-se o teste de regressão de Pearson ( $r$ ), no método Stewise correlation, no qual se selecionou somente as variáveis com significativa margem de contribuição na construção da equação, excluindo automaticamente, as variáveis com baixo nível de correlação. Observou-se como elemento crítico, adotando-se um nível de significância para $\mathrm{p}<0,05$, para a aceitação do modelo preditor.

No sentido de garantir a confiabilidade, separou-se a amostra em duas partes, $80 \%$ da amostra (48 sujeitos] foram utilizados na construção do modelo e os outros $20 \%$ [12 sujeitos], utilizados como base para ratificação do mesmo (amostra de confiabilidade].

Os participantes deste estudo foram separados segundo critérios randômicos, dizendo que as amostras sejam divididas para se determinar à validação ou a precisão da estimativa da formula desenvolvida. De acordo com os resultados apresentados na tabela 4 seguinte apresentam-se as variáveis selecionadas e seus níveis de correlação $r$ e $R^{2}$ (simples e coeficiente de determinação] e Erro Padrão Estimado [EPE].

Os níveis de correlação, e o nível de significância ficaram acima do esperado quando comparados aos modelos propostos pelos autores acima citados, isto se dá por alguns aspectos quanto características morfológicas e de treinabilidade da população amostral envolvida no experimento.

As informações geradas, segue abaixo a construção da tabela 5 , de validação para os resultados observados versus calculados cuja amostra constitui-se pelos os $20 \%$ selecionados anteriormente, onde a mesma pode ser simplificada no gráfico (figura 1).

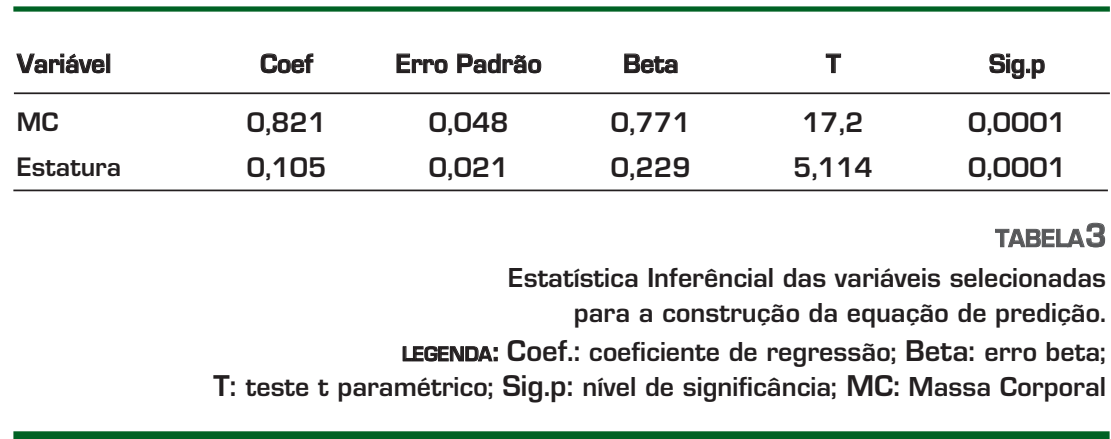

\section{DISCUSSÃO E CONCLUSÃO}

Os valores de 1RM, dos elementos que compuseram o desenvolvimento do modelo de predição ficaram abaixo da média quando confrontados aos de outros estudos ${ }^{5,18}$.

Em outra pesquisa o EPE não foi reportado, onde este seria de grande sustentação, pois utilizaram algumas variáveis antropométricas que constavam para o desenvolvimento desta equação ${ }^{16}$. Não foi comparado neste estudo o número de repetições realizados pelos participantes sendo este fator importante a ser levado em consideração, no que diz respeito à predição da força máxima ${ }^{14}$. Isto se deu pelo fato de que o objetivo deste estudo não foi desenvolver uma equação a partir do número máximo de repetições, mas sim pelo fato de utilizarem-se apenas variáveis antropométricas como critério de predição.

Os indicadores de relação se prenderam as variáveis antropométricas. para as variáveis de composição corporal, necessita-se de instrumental muito das vezes complexo e ha necessidade de conhecimento das técnicas de mensuração de dobras cutâneas. Tornando-se relevante a utilização de uma equação na qual se necessita apenas de uma balança com estadiômetro.

Quanto ao Erro Padrão Estimado (EPE), o modelo apresentado demonstra ter um menor erro estimado comparado aos de outros estudos $^{9,18}$. Ponto positivo neste achado é que,

\begin{tabular}{|c|c|c|c|}
\hline Estudo & Amostra & Exercício & Relações \\
\hline Dados do estudo & $M(n=60)$ & Supino reto & $\begin{array}{c}r=0,99, \text { sig } P<0,01 \\
R^{2}=0,98 E P E=3,378 \mathrm{~kg}\end{array}$ \\
\hline Kravitz $(2003)^{16}$ & $\begin{array}{c}H(n-18) \\
\text { adolescentes }\end{array}$ & Supino livre & não disponível \\
\hline $\begin{array}{l}\text { Ballman et al } \\
{\text { (1999 })^{3}}\end{array}$ & $M(n-124)$ & Supino livre & $\begin{array}{c}\mathrm{R}=0,51 \text { a } 0,63 \\
\mathrm{EPE}=5,3 \text { a } 5,7 \mathrm{~kg}\end{array}$ \\
\hline $\begin{array}{l}\text { Cummings e Finn } \\
(1998)^{9}\end{array}$ & $M(n-57)$ & Supino livre & $\begin{array}{l}\mathrm{r}=0,47 \mathrm{EPE}=2,3 \mathrm{~kg} \\
\text { Preditor circu. Braço }\end{array}$ \\
\hline Mayhew $(1999)^{18}$ & $M$ adolesc. (n-15) & Leg press & $\mathrm{R}=0,67 \mathrm{EPE}=20,2 \mathrm{~kg}$ \\
\hline
\end{tabular}




\begin{tabular}{cccc}
\hline Esperado & Calculado & Calc - erro & Calc + erro \\
\hline 64 & 71,1 & 67,7 & 74,5 \\
64 & 71 & 67,6 & 74,4 \\
64 & 66 & 62,6 & 69,3 \\
64 & 65,6 & 62,2 & 69 \\
64 & 67 & 63,6 & 70,4 \\
64 & 66,8 & 63,4 & 70,2 \\
63 & 65,1 & 61,8 & 68,5 \\
60 & 66,4 & 63 & 69,8 \\
60 & 68,1 & 64,7 & 71,5 \\
60 & 63,9 & 60,5 & 67,2 \\
60 & 64,1 & 60,7 & 67,5 \\
60 & 66,3 & 62,9 & 69,6 \\
\hline
\end{tabular}

TABELA5

Resultados Observados vs. Calculado.
De acordo com os resultados apresentados $^{3,9,16,18}$, na tabela 4 seguinte apresentam-se as variáveis selecionadas e seus níveis de correlação $r$ e $R^{2}$ (simples e coeficiente de determinação] e Erro Padrão Estimado (EPE), são equiparados aos apresentados pela literatura.

Desta forma, os resultados apresentados reportam a linearidade quanto aos valores de 1RM, reafirmando o critério das variáveis exclusas da equação gerada.

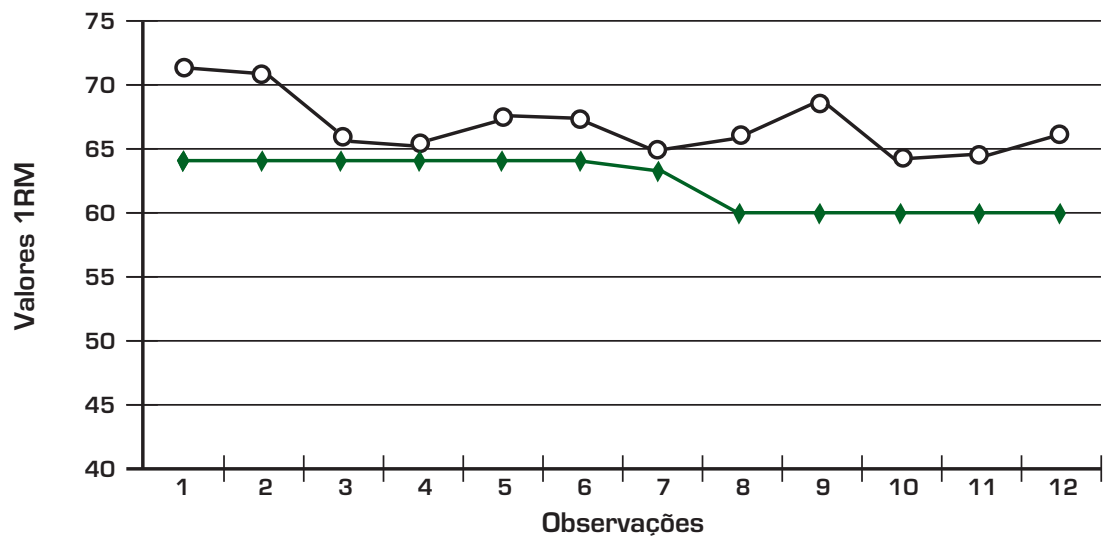

FIGURA1

Resultados Observados vs. Calculado.
Entretanto os resultados calculados e observados demonstram que pelo fato de cada elemento avaliado apresentar estaturas diferentes, este se torna o fator determinante, reafirmando o modelo encontrado, ou seja, indivíduos com o mesmo peso corporal, porém apresentando estaturas diferenciadas, podem demonstrar valores de força não equivalentes, sendo reafirmado pela variável da força relativa ${ }^{3,17}$.

\section{APLICAC̣ÕES PRÁTICAS}

Surge neste estudo à utilização da equação proposta, junto ao paradigma terminológico CTTP, preenchendo positivamente lacunas na confecção do trabalho com pesos para o exercício de supino reto, nas academias de musculação.

Cabe esclarecer que, a elaboração de uma equação de predição através de variáveis antropométricas, utilizando apenas uma balança e com estadiômetro, não necessitando da utilização de compassos de dobras cutâneas, paquímetros, etc., tornando-se mais rápido a mensuração da força máxima do indivíduo, desta forma otimizando a referência com vistas a elaboração de programas de musculação em alunos praticante de musculação recreativa em nível intermediários (mais de oito semanas de experiência], para o exercício proposto.

Mantendo a praticidade e a aplicabilidade do que foi desenvolvido, criou-se uma tabela (tabela 6), onde se conhecendo os valores da massa corporal e da estatura, identificam-se os valores máximos da força dinâmica no banco de supino horizontal com pesos livres. 
Revista de Desporto e Saúde

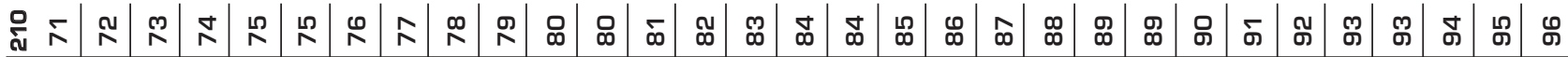
兽 兽六 荧卡 昰 峜 兽 号

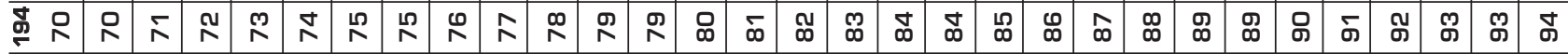

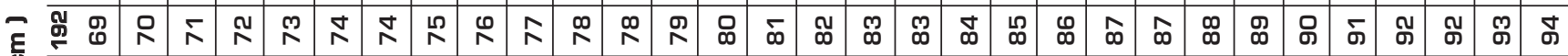
察旁 离

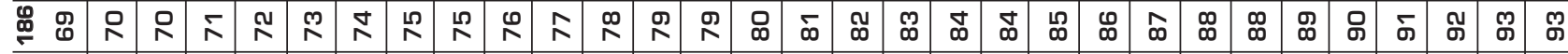
舟 家 品

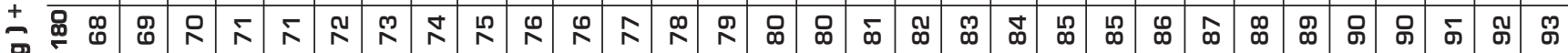

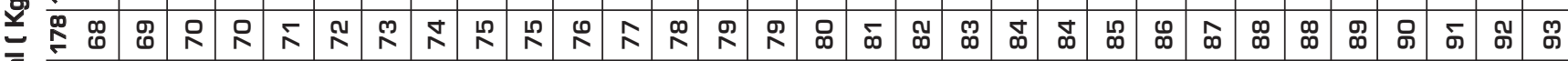

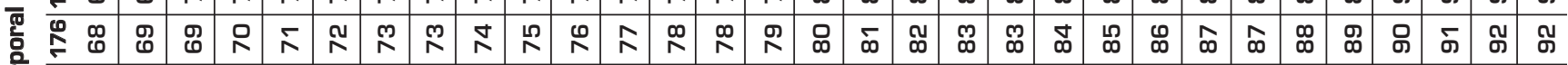

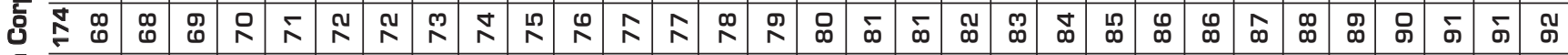

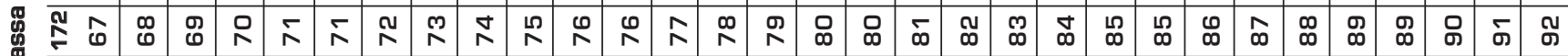

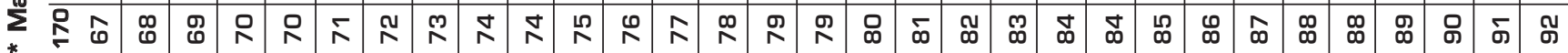

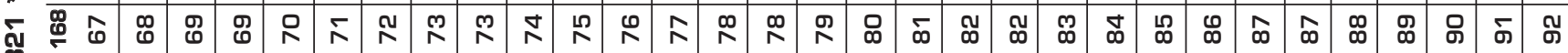
兽 㫐 䓽

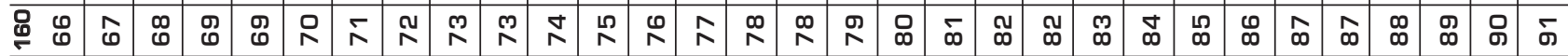

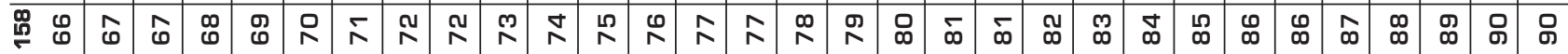

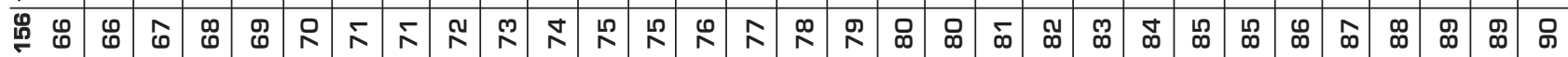
志 唒 总

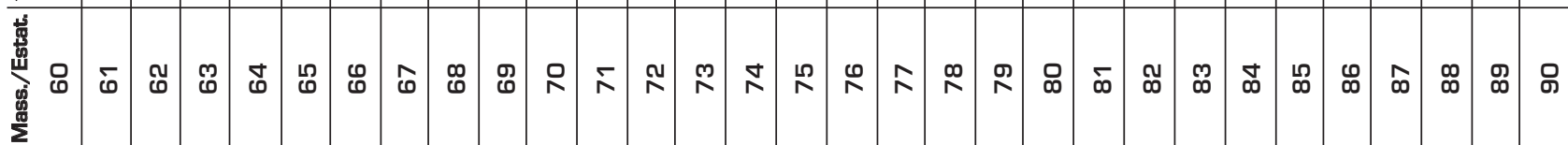

TABELA6

Predição da força dinâmica de membros superiores, através da massa corporal e estatura, para alunos intermediários no exercício de supino horizontal com o uso de peso livre. 


\begin{tabular}{l} 
CORRESPONDÊNCIA \\
Victor Machado Reis \\
UTAD - Departamento \\
de Ciências do Desporto \\
Apartado 1013 \\
5000 Vila Real - Portugal \\
E-mail: vreis@utad.pt \\
\hline REFERÊNCIAS \\
\hline
\end{tabular}

1. Adams GM. (1999) Exercise physiology - laboratory manual. Dubuque: Wm. C. Brown Communications Inc.

2. Baechle TR, Earle RW. (2000) Essential of strength training and conditioning. Champaign: Human Kinetics.

3. Ballmann KL, Scanlan JM, Mayhew JL, Lantz CD. (1999) Anthropometric dimensions to predict 1-RM bench press in untrained females. $J$ Sports Med Phys Fitness. 39(1):54-60.

4. Braith RW, Graves JE, Leggett SH, Pollock ML. (1993) Effect of training on the relationship between maximal and submaximal strength. Med Sci Sports Exerc. 25(1):132-8.

5. Chapman PP, Whiehead JR, Binkert RH. (1998) The 225 lbs reps-to-fatigue test as a submaximal estimate of 1 RM bench press performance in college football players. J Strength Cond Res. 12 (4): 258-261;

6. Chestnut JL, Docherty D. (1999) The effects of 4 and 10 repetition maximum weighttraining protocols on neuromuscular adaptations in untrained men. $J$ Strength Cond Res. 13(4):353-9.

7. Clarke DH. (1973) Adaptations in strength and muscular endurance resulting from exercise. Exer Sports Sci Rev. 1:73-102.
8. Cronin JB, Henderson ME. (2004) Maximal strength and power assessment in novice weight trainers. $J$ Strength Cond Res. 18(1):48-52.

9. Cummings $B$ and Finn KJ. (1998) Estimation of a one repetition maximum bench press for untrained women. J. Strength Cond Res. 12(4):262-265.

10. Fleck SJ and Kraemer WJ. (1999) Fundamentos do treinamento de força muscular. Porto Alegre: Ed. Artmed.

11. Gordon CC, Chumlea WC, Roche AF. (1988) Stature, recumbent length, and weight. In: Lohman TG, Roche AF, Martorell R. (editors). Anthropometric standardization reference manual. Champaign: Human Kinetics Books.

12. Häkkinen K, Alen M, Komi PV. (1995) Changes in isometric force and relaxation-time, electromyographic and muscle fibre characteristics of human skeletal muscle during strength training and detraining. Acta Physiol Scand. 125(4): 573-85.

13. Harris C, DeBeliso MA, SpitzerGibson TA, Adams KJ. (2004) The effect of resistance-training intensity on strength-gain response in the older adult. $J$ Strength Cond Res. 18(4):833-8.

14. Jackson AS and Pollock ML. (2004) Generalized equations for predicting body density of men. 1978. Br J Nutr. 91(1):161-8.

15. Kalapotharakos VI, Michalopoulos M, Tokmakidis SP, Godolias G, Gourgoulis V. (2005) Effects of a heavy and a moderate resistance training on functional performance in older adults. $J$ Strength Cond Res. 19(3):652-7

16. Kravitz L, Akalan C, Nowicki K, Kinzey SJ. (2003) Prediction of 1 repetition maximum in high-school power lifters. J Strength Cond Res. 17(5):167-172;
17. Mayhew JL, Piper FC and Ware JS. (1993) Anthropometric Correlates With Strength Performance Among Resistance Trained Athletes. $J$ Sports Med Phys Fitness. 33(2): 159-165.

18. Mayhew JL, Ware JS, Bemben MG, Wilt B, Ward TE, Farris B, Juraszez J, Slovak JP. (1999) The NFL-225 test a mensure of bench press strength in college foot-ball players. J Strength Cond Res. 13(2): 130-134.

19. Melanson EL, Sharp TA, Seagle HM, Donahoo WT, Grunwald GK, Peters JC, Hamilton JT, Hill JO. (2005) Twenty-four-hour metabolic responses to resistance exercise in women. $J$ Strength Cond Res. 19(1):61-6.

20. Ploutz-Snyder LL, Giamis EL. (2001) Orientation and familiarization to 1RM strength testing in old and young women. $J$ Strength Cond Res. 15(4):519-23.

21. Rikli RE, Jones CJ, Beam WC, Duncan SJ, Lamar B. (1996) Testing versus training effects on 1-RM strength assessment in older adults. Med Sci Sports Exerc. 28(5) Supplement:153.

22. Slaughter MH, Lohman TG, Boileau RA, Stillman RJ, Van Loan M, Horswill CA, Wilmore JH. (1984) Influence of maturation on relationship of skinfolds to body density: a cross-sectional study. Hum Biol. 56(4):681-9.

23. Siri WE. (1961) Body composition from fluid spaces and density: analysis of methods. In: Brozek J, Henschel A (ed). Techniques for measuring body composition. Washington: National Academy of Science.

24. Yu CC, Sung RY, So RC, Lui KC, Lau W, Lam PK, Lau EM. (2005) Effects of strength training on body composition and bone mineral content in children who are obese. J Strength Cond Res. 19(3):667-72. 